\title{
Left main coronary artery ostial disease: Prognostic role of the gap-angle ratio
}

\author{
Gianluca Rigatelli ${ }^{1 *} \mathbb{0}$, Marco Zuin ${ }^{2 *}$, Pavel Nikolov ${ }^{3}$, Dobrin Vassilev ${ }^{3}$ \\ ${ }^{1}$ Section of Cardiovascular and Endoluminal Interventions, Rovigo General Hospital, Rovigo, Italy \\ ${ }^{2}$ Department of Internal Medicine, University of Ferrara, School of Medicine, Ferrara, Italy \\ ${ }^{3}$ Department of Cardiology, Alexandrovska University, School of Medicine, Sofia, Bulgaria
}

Treatment of aorto-ostial coronary artery disease remains a challenge for interventional cardio logists due to the high rate of restenosis and stent misplacement often results in poor cardiovascular outcomes [1, 2]. Its anatomical position and plaque morphology may impact the rigidity of the lesion, possibly complicating the stenting procedure [3]. The gap-angle ratio (GAR) has been recently proposed as a method to describe the right coronary artery ostial stenosis rigidity and its impact on post-stenting outcomes [4]. We evaluated the use of the same parameter to describe isolated left main (LM) coronary artery ostial disease, analysing the prognostic role of GAR in these patients before stenting.

The clinical and instrumental records of 5435 consecutive patients underwent to coronary angiography in the documented institution were retrospectively analysed between January 2011 to January 2018 to identify patients with an isolated ostial LM disease and bypass surgery contraindications and/or refusal as determined by the local Heart Team. The local board approved the study.

A significant ostial LM lesion was defined as a lesion with $>50 \%$ diameter stenosis within $3 \mathrm{~mm}$ of the aortic ostium by quantitative coronary angiography analysis. Target lesion failure (TLF) was defined as the composite of cardiovascular death, target-vessel myocardial infarction, and clinically driven target lesion revascularization (TLR). For each patient we assessed the angle between the greater curvature of the aortic wall and LM take-off during both the diastolic (i.e. minimum angle) and systolic phases (i.e. maximum angle) in right anterior oblique $\left(30^{\circ}\right)$ position. The LM-GAR was calculated as follows:

$$
\frac{\text { Maximum angle-Mimimum angle }}{\text { Mimimum angle }}
$$

As evidenced in the formula (1), a high LM-GAR would indicate large motion of the LM ostium, similarly to the observations performed by Ohashi et al. [4]. Two independent cardiologists performed the measurement using the same software with an agreement of $98.3 \%$.

Stent repositioning after the first attempt and misplacement with need of a second stent were also recorded and analysed in each patient.

Information about the in-hospital outcome was obtained from an electronic clinical database for patients maintained at the documented institution and by reviewing hospital records for those discharged to referring hospitals. Post-discharge survival status was obtained from the Municipal Civil Registries.

Sixty-one patients were identified with isolated LM ostial stenosis: 7 were excluded due to a history of aortic valve replacement, 3 had concomitant significant lesions of the mid-left anterior descending or LM bifurcation, 2 had an aortitis and one was admitted due to a ST-segment elevation myocardial infarction. Finally, 47 patients ( 36 males, mean age $68.2 \pm 10.2$ years) were analysed (Table 1). During the procedure, patients who experienced stent repositioning or stent displacement

Address for correspondence: Prof. Gianluca Rigatelli, MD, PhD, FACP, FACC, FESC, FSCAI, Section of Adult Congenital Heart Disease Interventions, Cardiovascular Diagnosis and Endoluminal Interventions, Rovigo General Hospital, 45100 Rovigo, Italy, tel: +3903471912016, fax: +390425394513, e-mail: jackyheart71@yahoo.it

Received: 2.05.2020 Accepted: 24.10.2020

*Gianluca Rigatelli and Marco Zuin equally contributed to the study. 
Table 1. General characteristics of the patients enrolled, also stratified according the left main gap angle ratio (LM-GAR).

\begin{tabular}{|c|c|c|c|c|}
\hline & $\begin{array}{c}\text { All } \\
(n=47)\end{array}$ & $\begin{array}{l}\text { LM-GAR < } 0.23 \\
\quad(n=34)\end{array}$ & $\begin{array}{l}\text { LM-GAR } \geq 0.24 \\
\quad(n=13)\end{array}$ & $\mathbf{P}$ \\
\hline Age [years] & $68.2 \pm 10.2$ & $66.0 \pm 9.8$ & $70.4 \pm 11.3$ & 0.19 \\
\hline Males & $36(76.5 \%)$ & $26(76.7 \%)$ & $10(76.9 \%)$ & 0.98 \\
\hline Hypertension & $24(51.0 \%)$ & $18(52.9 \%)$ & $6(46.1 \%)$ & 0.67 \\
\hline Dyslipidemia & $24(51.0 \%)$ & $14(41.1 \%)$ & $10(76.9 \%)$ & 0.03 \\
\hline Diabetes mellitus & $20(42.5 \%)$ & $11(32.3 \%)$ & $9(69.2 \%)$ & 0.02 \\
\hline Previous smokers & $21(55.6 \%)$ & $14(41.1 \%)$ & $7(53.8 \%)$ & 0.43 \\
\hline Active smokers & $6(12.7 \%)$ & $4(11.7 \%)$ & $2(15.3 \%)$ & 0.74 \\
\hline Previous MI & $8(17.0 \%)$ & $5(14.7 \%)$ & $3(23.0 \%)$ & 0.50 \\
\hline Heart failure & $10(21.2 \%)$ & $7(20.5 \%)$ & $3(23.0 \%)$ & 0.85 \\
\hline $\mathrm{CKD}^{\circ}$ & $15(31.9 \%)$ & $8(23.5 \%)$ & $7(53.8 \%)$ & 0.05 \\
\hline COPD & $7(14.8 \%)$ & $5(14.7 \%)$ & $2(15.3 \%)$ & 0.97 \\
\hline Stroke & $7(14.8 \%)$ & $4(11.7 \%)$ & $3(23.0 \%)$ & 0.33 \\
\hline LVEF [\%] & $47.1 \pm 9.2$ & $46.2 \pm 8.7$ & $48.1 \pm 9.8$ & 0.55 \\
\hline CCS & $2.3 \pm 0.5$ & $2.3 \pm 0.9$ & $2.4 \pm 0.6$ & 0.71 \\
\hline SYNTAX score & $9.4 \pm 3.9$ & $9.4 \pm 3.6$ & $9.5 \pm 4.1$ & 0.94 \\
\hline Basal MLD [mm] & $1.7 \pm 1.1^{*}$ & $1.7 \pm 1.3^{*}$ & $1.8 \pm 1.0^{*}$ & 0.80 \\
\hline Final MLD [mm] & $4.3 \pm 0.5$ & $4.3 \pm 0.6$ & $4.4 \pm 0.3$ & 0.57 \\
\hline Lesion length [mm] & $12.1 \pm 3.6$ & $11.8 \pm 2.5$ & $12.3 \pm 3.5$ & 0.58 \\
\hline Orsiro $^{1}$ & $10(21.2 \%)$ & $7(20.5 \%)$ & $3(23.0 \%)$ & 0.85 \\
\hline Onyx ${ }^{2}$ & $25(53.1 \%)$ & $18(52.9 \%)$ & $7(53.8 \%)$ & 0.95 \\
\hline Promus Premier ${ }^{3}$ & $12(25.3 \%)$ & $9(26.4 \%)$ & $3(23.0 \%)$ & 0.81 \\
\hline 1-year TLF & $7(14.8 \%)$ & $2(5.8 \%)$ & $5(38.4 \%)$ & 0.005 \\
\hline 1-year CV mortality & $4(8.5 \%)$ & $1(2.9 \%)$ & $3(23.0 \%)$ & 0.03 \\
\hline Maximum angle ${ }^{\circ}$ & $103.6 \pm 9.8$ & $101.9 \pm 7.8$ & $105.4 \pm 13.2$ & 0.26 \\
\hline Minimum angle $^{\circ}$ & $83.3 \pm 10.5$ & $82.2 \pm 12.1$ & $84.4 \pm 8.6$ & 0.55 \\
\hline Angle gap ${ }^{\circ}$ & $20.3 \pm 9.2$ & $19.7 \pm 9.2$ & $21.0 \pm 9.6$ & 0.67 \\
\hline LM-GAR & $0.26 \pm 0.32$ & $0.11 \pm 0.60$ & $0.42 \pm 0.17$ & $<0.0001$ \\
\hline
\end{tabular}

Defined as an estimate glomerular filtration rate, using the CKD-EPI formula, $<60 \mathrm{~mL} / \mathrm{min} / \mathrm{m}^{2}$

${ }^{*} \mathrm{p}<0.0001$ between basal and final MLD after post dilatation in each group

'Biotronik Inc., Bulak, Switzerland; ${ }^{2}$ Medtronic Inc., Galway, Ireland; ${ }^{3}$ Boston Scientific Corp. Mantik, MA, USA

$\mathrm{MI}$ - myocardial infarction; CKD — chronic kidney disease; COPD — chronic obstructive pulmonary disease; LVEF — left ventricular ejection fraction; CCS - Canadian Cardiovascular Score; MLD - minimal lumen diameter; TLF — target lesion failure; CV — cardiovascular

resulting in need for a second stent (15 patients) had a mean LM-GAR angle higher than those without: $0.26 \pm 0.02$ vs. $0.21 \pm 0.03(\mathrm{p}=0.03)$.

On receiver operating characteristic analysis the optimal cut-off value for LM-GAR, as a predictor of 1 -year TLF was $\geq 0.24$ (area under curve of $0.86 \pm 0.5 ; 95 \%$ confidence interval [CI] 0.76-0.96, $\mathrm{p}=0.002$ ). At a mean follow-up of $11.9 \pm 0.6$ months, the rate of both TLF (38.4\% vs. $5.8 \%$, $\mathrm{p}=0.005)$ and cardiovascular mortality $(23.0 \%$ vs. $2.9 \%, \mathrm{p}=0.03$ ) were significantly higher in patients with an LM-GAR $\geq 0.24$. Sensitivity, specificity, positive predictive value and negative predictive value of LM-GAR for TLF were $81.8 \%$, $94.1 \%, 69.2 \%$, and 94.1, respectively. Mantel-Cox analysis revealed that there was a significant statistical difference in the occurrence of TLF after 1 year between patients with a LM-GAR $<0.23$ compared with those having a LM-GAR $\geq 0.24$ (log rank [Mantel-Cox] $\left.\chi^{2} 10.2, \mathrm{p}=0.01\right]$. Moreover, a multivariate Cox regression analysis demonstrated that 1-year TLF (hazard ratio [HR] 3.34, 95\% CI $2.29-3.77, \mathrm{p}=0.001$ ) were independently predicted by dyslipidaemia (HR 2.26, 95\% CI 1.89-2.46, $\mathrm{p}<0.001$ ) and diabetes mellitus (HR $1.86,95 \%$ CI $1.45-2.16, \mathrm{p}=0.03)$. 
The present results suggest that LM-GAR was able to predict the incidence of TLF and cardiovascular mortality at 1 year in patients with isolated LM ostial stenosis treated with percutaneous coronary intervention (PCI). Both the degree of steepness of the LM takeoff angle as well as extensive motion of the LM ostium are independent risk factors of adverse clinical events in the long-term period in these patients [1].

In the current study it was postulated that a high LM-GAR indicate large motion of the LM ostium which potentially can complicate stent placement and deployment resulting in stent malapposition or mild displacement. This hypothesis was confirmed by a statistically significant higher LM-GAR ratio in patients who experienced such problems during PCI.

High LM-GAR ratio seems to correlate with a potential increased challenge during the procedure and with less favourable outcomes at 1 year compared to patients with lower LM-GAR ratios: patients with a LM-GAR ratio $\geq 0.24$ require particular attention during PCI in order to overcome the large vessel motion, such as extra-backup guiding catheter and double wiring of the LM bifurcation.

Conflict of interest: None declared

\section{References}

1. Dishmon DA, Elhaddi A, Packard K, et al. High incidence of inaccurate stent placement in the treatment of coronary aortoostial disease. J Invasive Cardiol. 2011; 23(8): 322-326, indexed in Pubmed: 21828393.

2. Patel Y, Depta JP, Patel JS, et al. Impact of intravascular ultrasound on the long-term clinical outcomes in the treatment of coronary ostial lesions. Catheter Cardiovasc Interv. 2016; 87(2): 232-240, doi: 10.1002/ccd.25034, indexed in Pubmed: 23728924.

3. Gutiérrez-Chico JL, Villanueva-Benito I, Villanueva-Montoto L, et al. Szabo technique versus conventional angiographic placement in bifurcations $010-001$ of Medina and in aorto-ostial stenting: angiographic and procedural results. EuroIntervention. 2010; 5(7): 801-808, doi: 10.4244/eijv5i7a134, indexed in Pubmed: 20142194.

4. Ohashi K, Abe D, Kuroki N, et al. Clinical impact of the gap-angle ratio in patients with ostial lesions of the right coronary artery undergoing percutaneous coronary intervention. Heart Vessels. 2019; 34(11): 1717-1727, doi: 10.1007/s00380-019-01417-x, indexed in Pubmed: 31028408. 